\title{
COMPUTING BOUNDS FOR LINEAR FUNCTIONALS OF EXACT WEAK SOLUTIONS TO POISSON'S EQUATION
}

\author{
A. M. SAUER-BUDGE ${ }^{\dagger}$, J. BONET ${ }^{\ddagger}$, A. HUERTA ${ }^{\S}$, AND J. PERAIRE ${ }^{\dagger}$
}

\begin{abstract}
We present a method for Poisson's equation that computes guaranteed upper and lower bounds for the values of piecewise-polynomial linear functional outputs of the exact weak solution of the infinite-dimensional continuum problem with piecewise-polynomial forcing. The method results from exploiting the Lagrangian saddle point property engendered by recasting the output problem as a constrained minimization problem. Localization is achieved by Lagrangian relaxation and the bounds are computed by appeal to a local dual problem. The proposed method computes approximate Lagrange multipliers using traditional finite element approximations to calculate a primal and an adjoint solution along with well known hybridization techniques to calculate interelement continuity multipliers. The computed bounds hold uniformly for any level of refinement, and in the asymptotic convergence regime of the finite element method, the bound gap decreases at twice the rate of the energy norm measure of the error in the finite element solution. Given a finite element solution and its output adjoint solution, the method can be used to provide a certificate of precision for the output with an asymptotic complexity that is linear in the number of elements in the finite element discretization. The elemental contributions to the bound gap are always positive and hence lend themselves to be used as adaptive indicators, as we demonstrate with a numerical example.
\end{abstract}

AMS subject classifications. 65N15, 65N30, 49M29

1. Introduction. Uncertainty about the reliability of numerical approximations frequently undermines the utility of field simulations in the engineering design process: simulations are often not trusted because they lack reliable feedback on accuracy, or are more costly than necessary because they are performed with greater fidelity than necessary in an attempt to bolster trust. In addition to devitalized confidence, numerical uncertainty often causes ambiguity about the source of any discrepancies when using simulation results in concert with experimental measurements. Can the discretization error account for the discrepancies, or is the underlying continuum model inadequate?

While confidence in the precision of a field simulation can be buoyed by performing convergence studies, such studies are computationally very expensive and in practice are often not performed at more than a few conditions, if at all, due to cost and time constraints. For this reason, researchers and practitioners employ adaptive methods to converge the solution in a manner that costs less in time and resources than uniform refinement. Adaptive methods powered by current error estimation technology, however, provide only asymptotic guarantees of precision, at best, and no guarantees of precision, at worse, since the convergence of adaptive methods remains an open question [12].

Our observations of engineering practice inform us that integrated quantities such as forces and total fluxes are frequently queried quantitative outputs from field simulations and that design and analysis does not always require the full precision available. The primary objective of our method, therefore, is to certify the precision of integrated outputs for low fidelity simulations as well as high fidelity simulations. We call our bounds uniform to differentiate our goal of obtaining quantitative bounds for all levels of refinement from the lesser goal of obtaining quantitative bounds only asymptotically in the limit of refinement. In this regard, the complete procedure

\footnotetext{
${ }^{\dagger}$ Department of Aeronautics and Astronautics, Massachusetts Institute of Technology, USA

¥Department of Civil Engineering, University of Wales Swansea, UK

$\S$ Departamento de Matematica Aplicada III, Universitat Politecnica de Catalunya, Spain
} 
can be viewed as a polynomial time algorithm in the number of mesh elements that provides a certificate of precision for a predicted output. The certificate guarantees a minimum level of precision in the output from a particular finite-dimensional approximation with respect to the output from the infinite-dimensional model that it is approximating. Furthermore, the procedure provides local information that can be used in conjunction with adaptive meshing to efficiently drive a solution to an arbitrary and guaranteed precision.

Verification and a posteriori error analysis have a long history in the development of the finite element method with many different approaches forwarded and investigated. Ainsworth and Oden give a detailed overview of many of the approaches in [2]. Conceptually, our method descends from a long line of complementary energy methods beginning in the early 1970s when Fraeijs de Veubeke [7] proposed verifying the precision of a simulation by comparing the energy computed from a global primal approximation with the complementary energy computed from a global dual approximation. Global primal-dual methods offer a rich context for approximation, but suffer from the delicate nature of the global dual approximation, relatively high cost, and for verification, from a lack of relevant measure because the upper and lower bounding properties hold only for the total energy.

Much more closely related to our work are the works of Ladevèze [10, 9], Ainsworth and Oden [1], and of Destuynder [6], all of which consider local complementary energy problems for developing estimates for the energy norm of the error. In contrast to the work of Ladevèze, we endeavor to compute uniformly guaranteed two-sided bounds on an output, not an estimate of the error in an abstract norm. While the work of Ainsworth and Oden as well as the related work of Cao, Kelly and Sloan [5] require the exact solution of infinite-dimensional local problems in order to guarantee bounds, our method guarantees bounds uniformly with the solution of a finite-dimensional local problem. Our method differs from that of Destuynder in that it is not burdened with the explicit construction of globally conforming approximations to dual admissible vector fields. The work we present here extends earlier work done by Patera, Paraschivoiu, and Peraire $[14,13]$ on two-level residual based techniques for computing output bounds.

In this paper, we focus on the overarching structure of the method and do not consider the details of its implementation, nor extensions to non-piecewise-polynomial forcing or curved domains, nor more general equations such as nonsymmetric dissipative operators, which will be presented in future publications [17]. Section 2 presents the core concepts in the simpler setting of energy bounds, where the method has a clear variational meaning and a direct relationship to hybrid methods. Section 3 recasts the energy bound method as a method for linear functional output bounds, simultaneously carrying out an explicit extension to more relevant error measures and an implicit extension to nonvariational problems. Finally, the last section demonstrates the method with numerical results for three example problems. The last of the three examples shows how the method can be used to drive an adaptive refinement process.

1.1. Poisson's Equation. We consider Poisson's equation posed on polygonal domains, $\Omega$, in $d$ spacial dimensions and, only for the sake of simplicity of presentation, homogeneous Dirichlet boundaries, $\Gamma=\partial \Omega$. The Poisson problem is formulated weakly as: find $u \in \mathcal{U}$ such that

$$
\int_{\Omega} \nabla u \cdot \nabla v \mathrm{~d} \Omega=\int_{\Omega} f v \mathrm{~d} \Omega, \quad \forall v \in \mathcal{U},
$$


where $\mathcal{U}(\Omega) \equiv\left\{u \in \mathcal{H}^{1}(\Omega)|u|_{\Gamma}=0\right\}$ and the domain $\Omega$ is assumed when otherwise unspecified, that is, $\mathcal{U} \equiv \mathcal{U}(\Omega)$. As a consequence of all the Dirichlet boundaries being homogeneous, $\mathcal{U}$ serves as both the function set and test space in our presentation. While we present the method for homogeneous Dirichlet data, it can be easily extended to nonhomogeneous data and Neumann boundary conditions.

2. Computing Energy Bounds. We begin by developing a lower bound on the total energy of the system, $\frac{1}{2} \int_{\Omega} \nabla u \cdot \nabla u \mathrm{~d} \Omega-\int_{\Omega} f u \mathrm{~d} \Omega$, which in the context of heat conduction, combines the heat dissipation energy, $\frac{1}{2} \int_{\Omega} \nabla u \cdot \nabla u \mathrm{~d} \Omega$, and the potential energy of the thermal loads, $-\int_{\Omega} f u \mathrm{~d} \Omega$. There is a well known physical principle at work in this problem, related to the symmetric positive definite nature of the diffusion operator, which states that the solution, $u$, is the function that minimizes the total energy with respect to all other candidates in $\mathcal{U}$

$$
u=\arg \inf _{w \in \mathcal{U}} \frac{1}{2} \int_{\Omega} \nabla w \cdot \nabla w \mathrm{~d} \Omega-\int_{\Omega} f w \mathrm{~d} \Omega,
$$

as can easily be verified by comparing the Euler-Lagrange equation of this minimization statement to Poisson's equation (1.1). This minimization formulation makes it clear that if we look for a discrete approximation of (1.1) in a finite set of conforming functions, $\mathcal{U}_{h}$, for which $\mathcal{U}_{h} \subset \mathcal{U}$, then the resulting total energy predicted by the approximation will approach the exact value from above.

While insightful, this upper bound on the total energy has limited usefulness for two primary reasons. First, only rarely will the total energy be relevant to the purpose of solving the original problem. Second, even when it is relevant, the upper bound will most likely not be helpful for managing approximation uncertainty. In an engineering design task, the upper bound usually corresponds to the "best case scenario," as opposed to the "worst case scenario" which would be required to ensure feasibility of the design.

Our strategy for obtaining lower bounds on the energy in a cost efficient manner is to first decompose the global problem into independent local elemental subproblems by relaxing the continuity of the set $\mathcal{U}$ along edges of a triangular partitioning of $\Omega$, using approximate Lagrange multipliers, then accumulate the lower bound from the objective values of approximate local dual subproblems.

2.1. Weak Continuity Reformulation. We begin by partitioning the domain into a mesh, $\mathcal{T}_{h}$, of nonoverlapping open subdomains, $T$, called elements, for which $\bigcup_{T \in \mathcal{T}_{h}} \bar{T}=\bar{\Omega}$. We denote by $\partial T$ the edges, $\gamma$, constituting the boundary of a single element $T$, and by $\partial \mathcal{T}_{h}$ the network of all edges in the mesh. We have not yet evoked a discretization of $\mathcal{U}$, but merely a domain decomposition represented by a mesh. With the broken space

$$
\hat{\mathcal{U}} \equiv\left\{v \in L^{2}(\Omega)|v|_{T} \in \mathcal{H}^{1}(T), \forall T \in \mathcal{T}_{h}\right\},
$$

in which the continuity of $\mathcal{U}$ is broken across the mesh edges, $\partial \mathcal{T}_{h}$, we can reformulate the energy minimization statement (2.1) by explicitly enforcing continuity

$$
\begin{aligned}
u=\arg \inf _{\hat{w} \in \hat{\mathcal{U}}} & \frac{1}{2} \int_{\Omega} \nabla \hat{w} \cdot \nabla \hat{w} \mathrm{~d} \Omega-\int_{\Omega} f \hat{w} \mathrm{~d} \Omega \\
\text { s.t. } & \sum_{T \in \mathcal{T}_{h}} \int_{\partial T} \sigma_{T} \lambda \hat{w} \mathrm{~d} \Gamma=0, \quad \forall \lambda \in \Lambda,
\end{aligned}
$$


where, for $T, T_{\mathrm{N}} \in \mathcal{T}_{h}$ and an arbitrary ordering of the elements,

$$
\sigma_{T}(x)= \begin{cases}-1 & x \in \bar{T} \cap \bar{T}_{\mathrm{N}}, T<T_{\mathrm{N}} \\ +1 & \text { otherwise. }\end{cases}
$$

Integrals over the broken domain, such as $\int_{\Omega} \nabla \hat{w} \cdot \nabla \hat{v} \mathrm{~d} \Omega$, are understood as sums of integrals over the subdomains, such as $\left.\left.\sum_{T \in \mathcal{T}_{h}} \int_{T} \nabla \hat{w}\right|_{T} \cdot \nabla \hat{v}\right|_{T} \mathrm{~d} \Omega$. As there is no ambiguity, we have suppressed the trace operators from our notation for the boundary integrals to simplify the appearance of the expressions.

To see how the constraint arises, consider a single edge, $\gamma \in \partial \mathcal{T}_{h}$, with neighboring elements $T$ and $T_{\mathrm{N}}$, for which a strong continuity constraint can be written roughly as $\left.\hat{w}\right|_{T, \gamma}-\left.\hat{w}\right|_{T_{\mathrm{N}}, \gamma}=0$ on $\gamma$. An integral weak representation is obtained by multiplying by an arbitrary test function, $\lambda_{\gamma}$, taken from an appropriate space, $\Lambda(\gamma)$, integrating along the edge, and ensuring the resulting integrated quantity is zero for all possible test functions: $\int_{\gamma}\left(\left.\hat{w}\right|_{T, \gamma}-\left.\hat{w}\right|_{T_{\mathrm{N}}, \gamma}\right) \lambda_{\gamma} \mathrm{d} \Gamma=0, \quad \forall \lambda_{\gamma} \in \Lambda(\gamma)$. The constraint used above is obtained by re-writing the combination of all edge constraints as a combination of elemental contributions, using $\sigma_{T}$ to track the sign of the contribution. Since $\left.\hat{w}\right|_{T}$ is a member of $\mathcal{H}^{1}(T)$, the trace of $\left.\hat{w}\right|_{T}$ on an edge $\gamma$ is a member of $\mathcal{H}^{\frac{1}{2}}(\partial T)$. Therefore, $\lambda$ on $\gamma$ is a member of the dual of the trace space, $\mathcal{H}^{-\frac{1}{2}}(\gamma)$, and the continuity multiplier space $\Lambda$ is the corresponding product space taken over all the edges of the mesh.

Notice that we have relaxed the Dirichlet boundary conditions as well as the interior continuity. The homogeneous Dirichlet conditions are weakly enforced implicitly by the continuity constraint. We shall not prove it here, but it is important to know that the minimizer of the constrained minimization problem (2.3) is indeed $u$, the exact solution of Poisson's equation (1.1) [2, 4].

2.2. Localization by Continuity Relaxation. Considering the Lagrangian of the constrained minimization (2.3),

$$
\mathcal{L}(\hat{w} ; \lambda) \equiv \frac{1}{2} \int_{\Omega} \nabla \hat{w} \cdot \nabla \hat{w} \mathrm{~d} \Omega-\int_{\Omega} f \hat{w} \mathrm{~d} \Omega-\sum_{T \in \mathcal{T}_{h}} \int_{\partial T} \sigma_{T} \lambda \hat{w} \mathrm{~d} \Gamma
$$

we recall from the saddle point property of Lagrange multipliers and the strong duality of convex minimizations that for all $\tilde{\lambda} \in \Lambda$ there exists a lower energy bound, $\varepsilon^{-}$, satisfying

$$
\varepsilon^{-} \leq \inf _{\hat{w} \in \hat{\mathcal{U}}} \mathcal{L}(\hat{w} ; \tilde{\lambda}) \leq \sup _{\lambda \in \Lambda} \inf _{\hat{w} \in \hat{\mathcal{U}}} \mathcal{L}(\hat{w} ; \lambda)=\inf _{\hat{w} \in \hat{\mathcal{U}}} \sup _{\lambda \in \Lambda} \mathcal{L}(\hat{w} ; \lambda)=\varepsilon,
$$

where the value at optimality is the minimum total energy of the continuum system, $\varepsilon=\frac{1}{2} \int_{\Omega} \nabla u \cdot \nabla u \mathrm{~d} \Omega-\int_{\Omega} f u \mathrm{~d} \Omega$. The lower bounding minimization for a given $\tilde{\lambda}$ is separable, an important property allowing us to treat each element independently. In order to obtain a lower bound, $\tilde{\lambda}$ cannot be chosen arbitrarily. We obtain $\tilde{\lambda}$ by approximating the problem using finite elements in a manner that guarantees the relaxed minimization is bounded from below.

2.2.1. Continuity Multiplier Approximation. We now introduce the finite element approximation of Poisson's equation (1.1) as means of obtaining an approximate Lagrange multiplier. We first solve the finite-dimensional Poisson problem: find $u_{h} \in \mathcal{U}_{h}$ such that

$$
\int_{\Omega} \nabla u_{h} \cdot \nabla v \mathrm{~d} \Omega=\int_{\Omega} f v \mathrm{~d} \Omega, \quad \forall v \in \mathcal{U}_{h}
$$


where $\mathcal{U}_{h} \equiv\left\{v \in \mathcal{U}|v|_{T} \in \mathbb{P}^{p}(T), \forall T \in \mathcal{T}_{h}\right\}$ for $\mathbb{P}^{p}(T)$ the space of polynomials on element $T$ (in $d$ spacial dimensions) with degree less than or equal to $p$. Along with $\mathcal{U}_{h}$, we introduce the broken discrete space $\hat{\mathcal{U}}_{h} \equiv\left\{v \in \hat{\mathcal{U}}|v|_{T} \in \mathbb{P}^{p}(T), \forall T \in \mathcal{T}_{h}\right\}$ and the companion discrete Lagrange multiplier space $\Lambda_{h} \equiv\left\{\lambda \in \Lambda|\lambda|_{\gamma} \in \mathbb{P}^{p}(\gamma), \forall \gamma \in \partial \mathcal{T}_{h}\right\}$, where $\mathbb{P}^{p}(\gamma)$ is the space of polynomials on element edge $\gamma$ (in $d-1$ spacial dimensions) with degree less than or equal to $p$.

Once we have obtained $u_{h}$, we solve the gradient condition of (2.5) to obtain $\lambda_{h}$ : find $\lambda_{h} \in \Lambda_{h}$ such that

$$
\sum_{T \in \mathcal{T}_{h}} \int_{\partial T} \sigma_{T} \lambda_{h} \hat{v} \mathrm{~d} \Gamma=\int_{\Omega} \nabla u_{h} \cdot \nabla \hat{v} \mathrm{~d} \Omega-\int_{\Omega} f \hat{v} \mathrm{~d} \Omega, \quad \forall \hat{v} \in \hat{\mathcal{U}}_{h}
$$

We call this the equilibration problem, and we call any compatible Lagrange multiplier "equilibrating," since the problem has a nonunique solution. In the context of hybrid methods [4], this continuity multiplier is often referred to as a hybrid flux. As mentioned previously, this particular choice for the Lagrange multiplier ensures a finite lower bound.

LEMMA 2.1. If a Lagrange multiplier $\lambda_{h} \in \Lambda_{h}$ satisfies the equilibration condition (2.7), then $\inf _{\hat{w} \in \hat{\mathcal{U}}} \mathcal{L}\left(\hat{w} ; \lambda_{h}\right)$ is bounded from below.

Proof. Recall that the null space for the Poisson operator is the one-dimensional space of constants, $\mathbb{P}^{0}$, and let $\hat{\mathbb{P}}^{0}=\prod_{T \in \mathcal{T}_{h}} \mathbb{P}^{0}(T)$ denote the null space of the broken operator. Considering $\hat{c} \in \hat{\mathbb{P}}^{0} \subset \hat{\mathcal{U}}_{h}$ in the equilibration problem (2.7) and that any $\hat{w} \in \hat{\mathcal{U}}$ can be represented as $\hat{w}^{\prime}+\hat{c}$ for $\hat{w}^{\prime} \in \hat{\mathcal{U}} \backslash \hat{\mathbb{P}}^{0}$, it is easily shown that $\mathcal{L}\left(\hat{w}^{\prime}+\hat{c} ; \lambda_{h}\right)=\mathcal{L}\left(\hat{w}^{\prime} ; \lambda_{h}\right)$. For the Poisson equation, equilibration ensures that null space of the operator does not cause the minimization to become unbounded below. The existence of a minimum now follows from the coercivity of the Poisson operator in $\hat{\mathcal{U}} \backslash \hat{\mathbb{P}}^{0}$.

While not part of the classical finite element problem set, the equilibration problem has been addressed a number of times and in a number of contexts in the finite element community, not the least of which is in the context of error estimation. For our implementation, we use a method due to Ladevèze $[9,2]$ which has an asymptotically linear computational cost in the number of mesh vertices.

2.3. Local Dual Subproblem. Now that we have successfully decomposed the global problem into local elemental subproblems and determined a suitable approximation $\lambda_{h}$ for our continuity multiplier tilde $\lambda$, we can write the lower bounding minimization induced by the Lagrange saddle point property as

$$
\inf _{\hat{w} \in \hat{\mathcal{U}}} \mathcal{L}(\hat{w} ; \tilde{\lambda})=\sum_{T \in \mathcal{T}_{h}} \inf _{w \in \mathcal{U}(T)} J_{T}(w)
$$

for

$$
J_{T}(w) \equiv \frac{1}{2} \int_{T} \nabla w \cdot \nabla w \mathrm{~d} \Omega-\int_{T} f w \mathrm{~d} \Omega-\int_{\partial T} \sigma_{T} \tilde{\lambda} w \mathrm{~d} \Gamma,
$$

and consider a representative minimization subproblem. The minimization subproblem simply corresponds to a Poisson problem of the type represented in equation (1.1) with Neumann boundary conditions posed on a single subdomain. We have done nothing to change the nature of original problem, but have only acted to decompose the global problem into a sequence of independent local problems. 
We do not require, and in general cannot compute, the exact minimum of the infinite-dimensional local subproblem, but we do require a lower bound for it and we proceed now to introduce the primary ingredient for obtaining this local lower bound.

Proposition 2.2. If we define the positive functional

$$
J_{T}^{c}(\mathbf{q}) \equiv \frac{1}{2} \int_{T} \mathbf{q} \cdot \mathbf{q} \mathrm{d} \Omega
$$

where $\mathbf{q} \in \mathcal{H}(\operatorname{div} ; T)$ and $\mathcal{H}(\operatorname{div} ; T) \equiv\left\{\mathbf{q} \mid \mathbf{q} \in\left(L^{2}(T)\right)^{d}, \nabla \cdot \mathbf{q} \in L^{2}(T)\right\}$ for a problem posed in $d$ spacial dimensions, then we have

$$
J_{T}(w) \geq-J_{T}^{c}(\mathbf{q}), \quad \forall w \in \mathcal{H}^{1}(T), \forall \mathbf{q} \in \mathcal{Q}(T),
$$

for the set of functions

$$
\begin{aligned}
\mathcal{Q}(T) \equiv\left\{\mathbf{q} \in \mathcal{H}(\operatorname{div} ; T) \mid \int_{T} \nabla \cdot \mathbf{q} v \mathrm{~d} \Omega-\int_{\partial T} \mathbf{q} \cdot \mathbf{n} v \mathrm{~d} \Gamma\right. \\
\left.=-\int_{T} f v \mathrm{~d} \Omega-\int_{\partial T} \sigma_{T} \tilde{\lambda} v \mathrm{~d} \Gamma, \quad \forall v \in \mathcal{H}^{1}(T)\right\}
\end{aligned}
$$

Proof. We begin by appealing to the following positive expression

$$
\frac{1}{2} \int_{T}(\mathbf{q}-\nabla w)^{2} \mathrm{~d} \Omega \geq 0
$$

for any $w \in \mathcal{H}^{1}(T)$ and any $\mathbf{q} \in \mathcal{Q}(T)$. This expression expands to

$$
\frac{1}{2} \int_{T} \mathbf{q} \cdot \mathbf{q} \mathrm{d} \Omega+\frac{1}{2} \int_{T} \nabla w \cdot \nabla w \mathrm{~d} \Omega-\int_{T} \mathbf{q} \cdot \nabla w \mathrm{~d} \Omega \geq 0
$$

in which we apply the Green's identity $-\int_{T} \mathbf{q} \cdot \nabla w \mathrm{~d} \Omega=\int_{T} \nabla \cdot \mathbf{q} w \mathrm{~d} \Omega-\int_{\partial T} \mathbf{q} \cdot \mathbf{n} w \mathrm{~d} \Gamma$ to obtain

$$
\frac{1}{2} \int_{T} \mathbf{q} \cdot \mathbf{q} \mathrm{d} \Omega+\frac{1}{2} \int_{T} \nabla w \cdot \nabla w \mathrm{~d} \Omega+\int_{T} \nabla \cdot \mathbf{q} w \mathrm{~d} \Omega-\int_{\partial T} \mathbf{q} \cdot \mathbf{n} w \mathrm{~d} \Gamma \geq 0 .
$$

The constraint included in the definition of $\mathcal{Q}(T)$ makes this expression equivalent to

$$
\frac{1}{2} \int_{T} \mathbf{q} \cdot \mathbf{q} \mathrm{d} \Omega+\frac{1}{2} \int_{T} \nabla w \cdot \nabla w \mathrm{~d} \Omega-\int_{T} f w \mathrm{~d} \Omega-\int_{\partial T} \sigma_{T} \tilde{\lambda} w \mathrm{~d} \Gamma \geq 0 .
$$

Identifying $J_{T}(w)$ and $J_{T}^{c}(\mathbf{q})$ we arrive at the desired expression for the local lower bound.

The best possible local lower bound can be obtained with the following maximization problem

$$
\sup _{\mathbf{q} \in \mathcal{Q}(T)}-J_{T}^{c}(\mathbf{q}) \leq \inf _{w \in \mathcal{U}(T)} J_{T}(w),
$$

for which we will obtained equality as a result of the convexity of $J_{T}$ and $J_{T}^{c}$. It is clear that we have derived a classic dual formulation ${ }^{1}$ for our local elemental minimization problem and essentially transformed a primal minimization problem into a dual feasibility problem. As we have alluded to earlier, the functional $J_{T}^{c}(\mathbf{q})$ is often called the complementary energy functional [16], when taken over the whole domain, $\Omega$, with a globally admissible complementary field.

\footnotetext{
${ }^{1}$ The classic derivation for the dual of the Poisson problem would begin by letting $\mathbf{q}=\nabla w$ (a statement of Fourier's law in the context of heat conduction) and proceed by eliminating $w$ from the problem.
} 
2.3.1. Subproblem Approximation. Significantly, we can make these subproblems computable by choosing an appropriate finite-dimensional set in which to search for $\mathbf{q}$. At the very least the set must be chosen so that the divergence of its functions contain the forcing function, $f$, in $T$ and the normal traces of its functions contain the approximate continuity multiplier, $\lambda_{h}$, on $\partial T$. In multiple dimensions, however, the polynomial approximation for the continuity multiplier will nullify any components of the set with nonpolynomial normal trace. Therefore, we choose the polynomial approximation subset

$$
\begin{aligned}
\mathcal{Q}_{h}(T) \equiv\left\{\mathbf{q} \in\left(\mathbb{P}^{q}(T)\right)^{d} \mid \int_{T} \nabla \cdot \mathbf{q} v \mathrm{~d} \Omega-\int_{\partial T} \mathbf{q} \cdot \mathbf{n} v \mathrm{~d} \Gamma\right. & \\
& \left.=-\int_{T} f v \mathrm{~d} \Omega-\int_{\partial T} \sigma_{T} \lambda_{h} v \mathrm{~d} \Gamma, \forall v \in \mathcal{H}^{1}(T)\right\},
\end{aligned}
$$

with $q \geq p$. As a consequence, the method as we have presented it is limited to forcing functions, $\left.f\right|_{T}$, that are members of the polynomial space $\mathbb{P}^{r}(T)$ for $q>r$ on each elemental domain. While in one dimension we gain no advantage in taking $q$ greater than $r+1$, in multiple dimensions we can do so in an attempt to sharpen the bounds. The interior constraint data, $f$, and the boundary constraint data, $\sigma_{T} \lambda_{h}$, cannot be chosen independently of each other, but must satisfy a compatibility condition in order to ensure solvability as manifest by the following lemma.

LEMma 2.3. Suppose the forcing function $\left.f\right|_{T}$ is a member of $\mathbb{P}^{r}(T)$ and that $\lambda_{h}$ satisfies (2.7), then there exists at least one dual feasible function, $\mathbf{q}$, that is a member of $\mathcal{Q}_{h}(T)$ for $q \geq p$ and $q>r$.

Proof. We begin by expressing $\mathbf{q}$, a member of $\left(\mathbb{P}^{q}(T)\right)^{d}$, as the combination $\mathbf{q}=\mathbf{q}_{D}+\mathbf{q}_{0}$, with $\mathbf{q}_{D}$ a normal boundary condition satisfying component, $\mathbf{q}_{D} \cdot \mathbf{n}=$ $\sigma_{T} \lambda_{h}$ on $\partial T$, and $\mathbf{q}_{0}$ a homogeneous normal boundary condition satisfying component, $\mathbf{q}_{0} \cdot \mathbf{n}=0$ on $\partial T$. With this lifting, we can write the feasibility constraint as

$$
-\int_{T} \nabla \cdot \mathbf{q}_{0} v \mathrm{~d} \Omega=\int_{T} f v \mathrm{~d} \Omega+\int_{T} \nabla \cdot \mathbf{q}_{D} v \mathrm{~d} \Omega .
$$

Recognizing the divergence operator on the left hand side, which maps $\left(\mathbb{P}^{q}(T)\right)^{d}$ into $\mathbb{P}^{q-1}(T)$, we note that we need only test against $v \in \mathbb{P}^{q-1}(T)$. Furthermore, finitedimensional linear equations are solvable if and only if the right hand side data lies in the range of the operator, which is orthogonal to the null space of the adjoint operator. The adjoint operator is easily found to be $\int_{T} \mathbf{q}_{0} \cdot \nabla v \mathrm{~d} \Omega$ which has the nullspace $v \in \mathbb{P}^{0}(T)$, and thus the right hand side data must be in $\mathbb{P}^{q-1}(T) \backslash \mathbb{P}^{0}(T)$.

To prove solvability, we need only to verify that the right hand side data is orthogonal to the constants, since the requirements that $q \geq p$ and $q>r$ ensure that the right hand side data is in $\mathbb{P}^{q-1}$. Choosing $v=$ const in the right hand side of the constraint, rewritten as

$$
\int_{T} f v \mathrm{~d} \Omega+\int_{T} \nabla \cdot \mathbf{q}_{D} v \mathrm{~d} \Omega=\int_{T} f v \mathrm{~d} \Omega-\int_{T} \mathbf{q}_{D} \cdot \nabla v \mathrm{~d} \Omega+\int_{\partial T} \sigma_{T} \lambda_{h} v \mathrm{~d} \Gamma,
$$

reveals the compatibility condition

$$
\int_{\partial T} \sigma_{T} \lambda_{h} \mathrm{~d} \Gamma=-\int_{T} f \mathrm{~d} \Omega
$$


which is satisfied by our choice for $\lambda_{h}$, as can be seen by choosing $\hat{v}=$ const on $T$ in the equilibration condition (2.7). The equilibration condition thus ensures that the constraint data is compatible and that there exists at least one $\mathbf{q}$ satisfying the constraint.

2.4. Energy Bound Procedure. In discussing the global procedure and its properties, we denote the global aggregate of independent elemental quantities by accenting them with a diacritical hat as we did for the global broken quantities, and we denote the aggregate of local functional forms by dropping the subscript $T$. In particular, $\hat{\mathcal{Q}}_{h}$ denotes the aggregate approximate dual function space, $\prod_{T \in \mathcal{T}_{h}} \mathcal{Q}_{h}(T)$, and $J^{c}(\hat{\mathbf{q}})$ the aggregate dual energy functional, $\sum_{T \in \mathcal{T}_{h}} J_{T}^{c}\left(\left.\mathbf{q}\right|_{T}\right)$. The complete method for the energy bounds consists of three steps:

1. Global Approximation: Find $u_{h} \in \mathcal{U}_{h}$ such that

$$
\int_{\Omega} \nabla u_{h} \cdot \nabla v \mathrm{~d} \Omega=\int_{\Omega} f v \mathrm{~d} \Omega, \quad \forall v \in \mathcal{U}_{h}
$$

and calculate the upper bound $\varepsilon_{h}^{+}=-\frac{1}{2} \int_{\Omega} \nabla u_{h} \cdot \nabla u_{h} \mathrm{~d} \Omega$.

2. Global Equilibration: Find $\lambda_{h} \in \Lambda_{h}$ such that

$$
\sum_{T \in \mathcal{T}_{h}} \int_{\partial T} \sigma_{T} \lambda_{h} \hat{v} \mathrm{~d} \Gamma=\int_{\Omega} \nabla u_{h} \cdot \nabla \hat{v} \mathrm{~d} \Omega-\int_{\Omega} f \hat{v} \mathrm{~d} \Omega, \quad \forall \hat{v} \in \hat{\mathcal{U}}_{h}
$$

3. Local Dual Approximations: Find $\varepsilon_{h}^{-}$such that

$$
\varepsilon_{h}^{-}=\sup _{\hat{\mathbf{q}}_{h} \in \hat{\mathcal{Q}}_{h}}-J^{c}\left(\hat{\mathbf{q}}_{h}\right)
$$

The last step requires the solution of a series of finite-dimensional quadratic programming problems with convex objective functions and linear equality constraints. The per-element cost remains low due to the small size of the elemental subproblems, while the total cost of computing the lower bound is asymptotically linear in the number elements.

2.4.1. Properties of the Energy Bound. As previously discussed, the upper bound follows directly from the conforming nature of the finite element approximation and the lower bound follows directly from Proposition 2.2. We close our presentation of the energy bound method by showing that the lower bound converges at the same rate as the upper bound, and thus inherits the well known a priori finite element convergence property for the energy norm of the error. We begin by proving an orthogonality result.

LEMMA 2.4. Let $\hat{\mathbf{p}}_{h}$ be any dual feasibility correction to $\nabla u_{h}$ such that $\hat{\mathbf{q}}_{h}=$ $\nabla u_{h}+\hat{\mathbf{p}}_{h}$ is a member of $\hat{\mathcal{Q}}_{h}$, then $\hat{\mathbf{p}}_{h}$ satisfies the orthogonality property

$$
\sum_{T \in \mathcal{T}_{h}} \int_{T} \hat{\mathbf{p}}_{h} \cdot \nabla \hat{v} \mathrm{~d} \Omega=0, \quad \forall \hat{v} \in \hat{\mathcal{U}}_{h}
$$

Proof. We begin by examining the condition that the feasibility correction $\hat{\mathbf{p}}_{h}$ must satisfy by substituting $\nabla u_{h}+\hat{\mathbf{p}}_{h}$ into the constraint contained in the definition 
of $\hat{\mathcal{Q}}_{h}$, summed over the elements, to obtain

$$
\begin{aligned}
\int_{\Omega} \nabla \cdot \hat{\mathbf{p}}_{h} \hat{v} \mathrm{~d} \Omega & -\sum_{T \in \mathcal{T}_{h}} \int_{\partial T} \hat{\mathbf{p}}_{h} \cdot \mathbf{n} \hat{v} \mathrm{~d} \Gamma=-\int_{\Omega} f \hat{v} \mathrm{~d} \Omega-\int_{\Omega} \nabla \cdot \nabla u_{h} \hat{v} \mathrm{~d} \Omega \\
& -\sum_{T \in \mathcal{T}_{h}} \int_{\partial T} \sigma_{T} \lambda_{h} \hat{v} \mathrm{~d} \Gamma+\sum_{T \in \mathcal{T}_{h}} \int_{\partial T} \nabla u_{h} \cdot \mathbf{n} \hat{v} \mathrm{~d} \Gamma, \quad \forall \hat{v} \in \hat{\mathcal{U}}_{h} .
\end{aligned}
$$

Applying Green's formula to both the $\hat{\mathbf{p}}_{h}$ and $u_{h}$ terms yields the equivalent constraint

$$
\int_{\Omega} \hat{\mathbf{p}}_{h} \cdot \nabla \hat{v} \mathrm{~d} \Omega=\int_{\Omega} f \hat{v} \mathrm{~d} \Omega-\int_{\Omega} \nabla u_{h} \cdot \nabla \hat{v} \mathrm{~d} \Omega+\sum_{T \in \mathcal{T}_{h}} \int_{\partial T} \sigma_{T} \lambda_{h} \hat{v} \mathrm{~d} \Gamma, \quad \forall \hat{v} \in \hat{\mathcal{U}}_{h}
$$

Restricting $\hat{v}$ to $\hat{\mathcal{U}}_{h}$ produces the sought orthogonality property as a consequence of equilibration (2.17).

Lemma 2.5. Let $\hat{\mathbf{p}}_{h}^{*}$ be the dual feasibility correction to $\nabla u_{h}$ that maximizes $-J^{c}\left(\hat{\mathbf{p}}_{h}\right)$ such that $\nabla u_{h}+\hat{\mathbf{p}}_{h}^{*}$ is a member of $\hat{\mathcal{Q}}_{h}$, then $J^{c}\left(\hat{\mathbf{p}}_{h}^{*}\right)$ is bounded from above by

$$
J^{c}\left(\hat{\mathbf{p}}_{h}^{*}\right) \leq C\left|u-u_{h}\right|_{1}^{2},
$$

for the semi-norm $|v|_{1}^{2} \equiv \int_{\Omega} \nabla v \cdot \nabla v \mathrm{~d} \Omega$, if the approximate continuity multiplier $\lambda_{h}$ computed in (2.17) has the bound

$$
\sum_{T \in \mathcal{T}_{h}} h^{\frac{1}{2}}\left\|\lambda-\lambda_{h}\right\|_{\partial T} \leq C\left|u-u_{h}\right|_{1}
$$

where $\left.\lambda\right|_{\partial T} \equiv \sigma_{T} \frac{\partial u}{\partial \mathbf{n}}$ is the exact continuity multiplier and $\|v\|_{\partial T}^{2} \equiv \int_{\partial T} v^{2} \mathrm{~d} \Gamma$. Everywhere, $C$ is a generic constant independent of $h=\operatorname{diam}(T)$.

Proof. Using the constraint $(2.21)$ and the definition $\hat{\mathcal{P}}_{h}=\prod_{T \in \mathcal{T}_{h}}\left(\mathbb{P}^{q}(T)\right)^{d}$, the

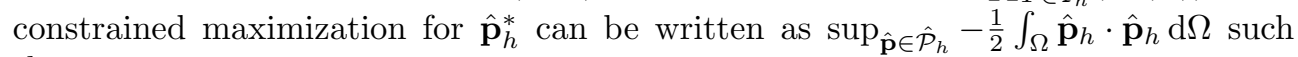
that

$$
-\int_{\Omega} \hat{\mathbf{p}}_{h} \cdot \nabla \hat{\phi} \mathrm{d} \Omega=\int_{\Omega} \nabla u_{h} \cdot \nabla \hat{\phi} \mathrm{d} \Omega-\int_{\Omega} f \hat{\phi} \mathrm{d} \Omega-\sum_{T \in \mathcal{T}_{h}} \int_{\partial T} \sigma_{T} \lambda_{h} \hat{\phi} \mathrm{d} \Gamma
$$

for all $\hat{\phi} \in \hat{\mathbb{P}}^{q+1}$. The gradient condition, informs us that $\hat{\mathbf{p}}_{h}^{*}=\nabla \hat{\phi}^{*}$. Since $\hat{\phi}^{*}$ is defined uniquely only up to a constant on each element because of compatibility, we can choose $\hat{\phi}^{*}$ to be of zero mean over each element, $\left.\int_{T} \hat{\phi}^{*}\right|_{T} \mathrm{~d} \Omega=0$.

The approximate solution $u_{h}$ has an associated approximate continuity multiplier $\lambda_{h}$ satisfying (2.17), while the exact solution $u$ also has an associated exact continuity multiplier $\lambda$ satisfying

$$
\sum_{T \in \mathcal{T}_{h}} \int_{\partial T} \sigma_{T} \lambda \hat{v} \mathrm{~d} \Gamma=\int_{\Omega} \nabla u \cdot \nabla \hat{v} \mathrm{~d} \Omega-\int_{\Omega} f \hat{v} \mathrm{~d} \Omega, \quad \forall \hat{v} \in \hat{\mathcal{U}}
$$

as can be verified by integration by parts. Adding (2.25) to the constraint of (2.24) 
with $\hat{\mathbf{p}}_{h}=\hat{\mathbf{p}}_{h}^{*}$ and $\hat{v}=\hat{\phi}^{*}$ we find for $\|\hat{v}\|^{2}=\sum_{T \in \mathcal{T}_{h}} \int_{T} v^{2} \mathrm{~d} \Omega$ that

$$
\begin{aligned}
\int_{\Omega} \hat{\mathbf{p}}_{h}^{*} \cdot \nabla \hat{\phi}^{*} \mathrm{~d} \Omega & =\int_{\Omega} \nabla\left(u-u_{h}\right) \cdot \nabla \hat{\phi}^{*} \mathrm{~d} \Omega-\sum_{T \in \mathcal{T}_{h}} \int_{\partial T} \sigma_{T}\left(\lambda-\lambda_{h}\right) \hat{\phi}^{*} \mathrm{~d} \Gamma \\
& \leq C\left|u-u_{h}\right|_{1}\left\|\nabla \hat{\phi}^{*}\right\|+\sum_{T \in \mathcal{T}_{h}} C\left\|\lambda-\lambda_{h}\right\|_{\partial T}\left\|\hat{\phi}^{*}\right\|_{\partial T} \\
& \leq C\left|u-u_{h}\right|_{1}\left\|\nabla \hat{\phi}^{*}\right\|+\sum_{T \in \mathcal{T}_{h}} C h^{\frac{1}{2}}\left\|\lambda-\lambda_{h}\right\|_{\partial T}\left\|\nabla \hat{\phi}^{*}\right\|,
\end{aligned}
$$

in which we applied the inequality $\|w\|_{\partial T} \leq C h^{\frac{1}{2}}|w|_{1, T}$, valid for any $w \in \mathcal{H}^{1}(T)$ that has zero mean [11]. Finally, after invoking the bound (2.23) we complete the proof by substituting $\nabla \hat{\phi}^{*}=\hat{\mathbf{p}}_{h}^{*}$, dividing both sides by $\left\|\hat{\mathbf{p}}_{h}\right\|$, and recognizing that $\left\|\hat{\mathbf{p}}_{h}^{*}\right\|^{2}=2 J^{c}\left(\hat{\mathbf{p}}_{h}^{*}\right)$.

Ainsworth and Oden prove in [1] that under certain assumptions the flux average of the finite element solution across the edges is bounded by (2.23) so that, by way of the triangle inequality, the burden rests in showing that the nonunique equilibrating corrections required to satisfy (2.17) decrease at the requisite rate. Maday and Patera give in [11] a basic method for computing approximate continuity multipliers that has been proven a priori to satisfy (2.23).

Proposition 2.6. Suppose that $\lambda_{h}$ is the solution of the equilibration problem (2.17) for $u_{h}$ the solution of the finite element approximation problem (2.16) then

$$
\varepsilon-\varepsilon^{-} \leq C\left|u-u_{h}\right|_{1}^{2}
$$

Proof. Let $\hat{\mathbf{p}}_{h}^{*}$ be chosen according to Lemma 2.5, then

$$
-J^{c}\left(\nabla u_{h}+\hat{\mathbf{p}}_{h}^{*}\right) \leq \sup _{\hat{\mathbf{q}}_{h} \in \hat{\mathcal{Q}}_{h}}-J^{c}\left(\hat{\mathbf{q}}_{h}\right)=-J^{c}\left(\hat{\mathbf{q}}_{h}^{*}\right),
$$

for $\hat{\mathbf{q}}_{h}^{*}=\arg \sup _{\hat{\mathbf{q}}_{h} \in \hat{\mathcal{Q}}_{h}}-J^{c}\left(\hat{\mathbf{q}}_{h}\right)$. From this relationship and from the definition of $\hat{\mathbf{p}}_{h}^{*}$ we know that $J^{c}\left(\hat{\mathbf{q}}_{h}^{*}\right) \leq J^{c}\left(\nabla u_{h}\right)+J^{c}\left(\hat{\mathbf{p}}_{h}^{*}\right)$, because $\sum_{T \in \mathcal{T}_{h}} \int_{T} \hat{\mathbf{p}}_{h}^{*} \cdot \nabla u_{h} \mathrm{~d} \Omega=0$ from Lemma 2.4 and the fact that $u_{h}$ is a member of $\hat{\mathcal{U}}_{h}$. Adding the exact energy $\varepsilon=-J^{c}(\nabla u)$ to each side and recalling that $\varepsilon_{h}^{+}=-J^{c}\left(\nabla u_{h}\right)$ and $\varepsilon_{h}^{-}=-J^{c}\left(\hat{\mathbf{q}}_{h}^{*}\right)$ we have our desired result

$$
\varepsilon-\varepsilon_{h}^{-} \leq \varepsilon-\varepsilon_{h}^{+}+J^{c}\left(\hat{\mathbf{p}}_{h}^{*}\right) \leq C\left|u-u_{h}\right|_{1}^{2},
$$

where we have again evoked Lemma 2.5 in addition to the well known finite element energy error bound.

3. Computing Output Bounds. We will continue to keep the presentation simple by considering only simple linear functional interior outputs. In particular, we will develop upper and lower bounds, $s^{ \pm}$, on the output quantity

$$
s \equiv \int_{\Omega} f^{\mathcal{O}} u \mathrm{~d} \Omega
$$

where $u$ is the exact solution of Poisson's equation (1.1) and $\left.f^{\mathcal{O}}\right|_{T}$ is a member of $\mathbb{P}^{r}(T)$ for all elements $T$ in $\mathcal{T}_{h}$. We stress, however, that more interesting outputs, such as boundary fluxes, can also be treated using techniques previously employed in the context of two-level methods (see, for example, the treatment of the normal force output for linear elasticity in [14]). 
3.1. Weak Continuity Reformulation. To begin, we must formulate a generalized analogue to the minimization statement (2.3). There are two parts to this task. First, we must replace the intrinsic energy of the variational problem with an energy reformulation of the linear output functional. Second, now that the minimization of the objective functional no longer corresponds to the solution of our original equation, we must explicitly ensure that the minimizer is the solution to our problem by including it as a constraint. Furthermore, to obtain both upper and lower bounds, we consider two cases which vary by the sign of the original output. The resulting pair of constrained minimization statements for the homogeneous ${ }^{2}$ Dirichlet boundary problem under consideration are

$$
\begin{array}{ccc}
\mp s=\inf _{\hat{w}^{ \pm} \in \hat{\mathcal{U}}} \mp \int_{\Omega} f^{\mathcal{O}} \hat{w}^{ \pm} \mathrm{d} \Omega+\frac{\kappa}{2}\left\{\int_{\Omega} \nabla \hat{w}^{ \pm} \cdot \nabla\left(\hat{w}^{ \pm}-\bar{u}\right) \mathrm{d} \Omega-\int_{\Omega} f\left(\hat{w}^{ \pm}-\bar{u}\right) \mathrm{d} \Omega\right\} \\
\text { s.t. } \quad & \int_{\Omega} \nabla \hat{w}^{ \pm} \cdot \nabla \psi \mathrm{d} \Omega=\int_{\Omega} f \psi \mathrm{d} \Omega, & \forall \psi \in \mathcal{U}, \\
\sum_{T \in \mathcal{T}_{h}} \int_{\partial T} \sigma_{T} \lambda \hat{w}^{ \pm} \mathrm{d} \Gamma=0, & \forall \lambda \in \Lambda,
\end{array}
$$

where $\bar{u}$ is any element of space $\mathcal{U}$, and $\kappa$ is a positive real scaling parameter which serves both as a coefficient providing dimensional consistency in the engineering context and as an additional degree of freedom which we will use to tighten the bounds. The quadratic objective functional has been constructed so that all terms but the desired output functional vanish when $\hat{w}^{ \pm}$is the exact solution, $u$, while the constraints enforce equilibrium and interelement continuity.

Paraschivoiu, Peraire and Patera [14, 13] originally proposed this reformulation in the context of two-level output bounding methods which appeal to a second refined but localized finite element approximation and therefore provided bounds only against a refined finite element approximation instead of the exact infinite-dimensional solution. With this constrained minimization reformulation, we can proceed more or less mechanically to apply the ideas from the energy bound to this more general context. The development of the output bound is very close to that for the energy bound, but with the extra burden of carrying an additional Lagrange multiplier for the equilibrium constraint and of managing the concurrent development of both upper and lower bounds on the output, as neither arise implicitly from the finite element discretization.

3.2. Localization by Continuity Relaxation. Considering the Lagrangian of problem (3.2),

$$
\begin{aligned}
\mathcal{L}^{ \pm}\left(\hat{w}^{ \pm} ; \psi^{ \pm}, \lambda^{ \pm}\right) & \\
\equiv & \mp \int_{\Omega} f^{\mathcal{O}} \hat{w}^{ \pm} \mathrm{d} \Omega+\frac{\kappa}{2}\left\{\int_{\Omega} \nabla \hat{w}^{ \pm} \cdot \nabla\left(\hat{w}^{ \pm}-\bar{u}\right) \mathrm{d} \Omega-\int_{\Omega} f\left(\hat{w}^{ \pm}-\bar{u}\right) \mathrm{d} \Omega\right\} \\
& +\int_{\Omega} f \psi^{ \pm} \mathrm{d} \Omega-\int_{\Omega} \nabla \hat{w}^{ \pm} \cdot \nabla \psi^{ \pm} \mathrm{d} \Omega-\sum_{T \in \mathcal{T}_{h}} \int_{\partial T} \sigma_{T} \lambda^{ \pm} \hat{w}^{ \pm} \mathrm{d} \Gamma
\end{aligned}
$$

\footnotetext{
${ }^{2}$ The extension to nonhomogeneous Dirichlet boundaries requires choosing $\bar{u}$ from the set of admissible functions and weakly enforcing the Dirichlet boundary data, $u_{D}$, by replacing the continuity constraint with $\sum_{T \in \mathcal{T}_{h}} \int_{\partial T} \sigma_{T} \lambda \hat{w}^{ \pm} \mathrm{d} \Gamma=\sum_{\gamma \in \partial \mathcal{T}_{h}} \int_{\gamma} \sigma_{T(\gamma)} \lambda u_{D} \mathrm{~d} \Gamma, \forall \lambda \in \Lambda$.
} 
we know, as we did for the energy bound, from the saddle point property of Lagrange multipliers and from the strong duality of convex minimizations that

$$
\inf _{\hat{w^{ \pm} \in \hat{\mathcal{U}}}} \mathcal{L}^{ \pm}\left(\hat{w}^{ \pm} ; \tilde{\psi}^{ \pm}, \tilde{\lambda}^{ \pm}\right) \leq \sup _{\substack{\psi^{ \pm} \in \mathcal{U} \\ \lambda \in \Lambda}} \inf _{\hat{w} \pm \hat{\mathcal{U}}} \mathcal{L}^{ \pm}\left(\hat{w}^{ \pm} ; \psi^{ \pm}, \lambda^{ \pm}\right)=\mp s .
$$

for all $\left(\tilde{\psi}^{ \pm}, \tilde{\lambda}^{ \pm}\right) \in \mathcal{U} \times \Lambda$. The lower bounding minimization for a given $\tilde{\lambda}^{ \pm}$and $\tilde{\psi}^{ \pm}$is separable and, for an appropriate choice for $\tilde{\lambda}^{ \pm}$, provides nontrivial upper and lower bounds on the exact output $s$.

3.2.1. Lagrange Multiplier Approximation. We proceed, as we did for the energy bound, to obtain approximate Lagrange multipliers with a finite element discretization of the gradient condition of Equation (3.3). Let $\psi_{h}^{ \pm}= \pm \psi_{h}, \lambda_{h}^{ \pm}=\frac{\kappa}{2} \lambda_{h}^{u} \pm \lambda_{h}^{\psi}$, and $\bar{u}=u_{h}$, all of which we find by solving the following discrete problems

1. Find $u_{h} \in \mathcal{U}_{h}$ such that

$$
\int_{\Omega} \nabla u_{h} \cdot \nabla v \mathrm{~d} \Omega=\int_{\Omega} f v \mathrm{~d} \Omega, \quad \forall v \in \mathcal{U}_{h},
$$

2. Find $\psi_{h} \in \mathcal{U}_{h}$ such that

$$
\int_{\Omega} \nabla v \cdot \nabla \psi_{h} \mathrm{~d} \Omega=-\int_{\Omega} f^{\mathcal{O}} v \mathrm{~d} \Omega, \quad \forall v \in \mathcal{U}_{h},
$$

3. Find $\lambda_{h}^{u} \in \Lambda_{h}$ such that

$$
\sum_{T \in \mathcal{T}_{h}} \int_{\partial T} \sigma_{T} \lambda_{h}^{u} \hat{v} \mathrm{~d} \Gamma=\int_{\Omega} \nabla u_{h} \cdot \nabla \hat{v} \mathrm{~d} \Omega-\int_{\Omega} f \hat{v} \mathrm{~d} \Omega, \quad \forall \hat{v} \in \hat{\mathcal{U}}_{h}
$$

4. Find $\lambda_{h}^{\psi} \in \Lambda_{h}$ such that

$$
\sum_{T \in \mathcal{T}_{h}} \int_{\partial T} \sigma_{T} \lambda_{h}^{\psi} \hat{v} \mathrm{~d} \Gamma=-\int_{\Omega} f^{\mathcal{O}} \hat{v} \mathrm{~d} \Omega-\int_{\Omega} \nabla \hat{v} \cdot \nabla \psi_{h} \mathrm{~d} \Omega, \quad \forall \hat{v} \in \hat{\mathcal{U}}_{h}
$$

The first two problems comprise the well known primal-adjoint pair which occur often in output oriented a posteriori error estimation techniques $[3,14,13]$ as well as in computational approaches to design optimization [8], while the last two problems are their independent equilibrations. The first and third problems are identical to the global approximation problems required for the energy bound. These particular choices for the Lagrange multipliers ensure a finite lower bound in the saddle point property.

LEMma 3.1. If the Lagrange multipliers $\psi_{h}^{ \pm}= \pm \psi_{h}$ and $\lambda_{h}^{ \pm}=\frac{\kappa}{2} \lambda_{h}^{u} \pm \lambda_{h}^{\psi}$ satisfy the equilibration conditions (3.6) and (3.7), then the minimums $\inf _{\hat{w}^{ \pm} \in \hat{\mathcal{U}}} \mathcal{L}\left(\hat{w}^{ \pm} ; \psi_{h}^{ \pm}, \lambda_{h}^{ \pm}\right)$ are bounded from below.

Proof. This is true for essentially the same reason that it is true for Lemma 2.1. The only algebraic difference being that in the present output bounding case the property $\mathcal{L}^{ \pm}\left(\hat{w}^{ \pm^{\prime}}+\hat{c} ; \psi_{h}^{ \pm}, \lambda_{h}^{ \pm}\right)=\mathcal{L}\left(\hat{w}^{ \pm^{\prime}} ; \psi_{h}^{ \pm}, \lambda_{h}\right)$ results from the combined action of both equilibration conditions.

3.3. Local Dual Subproblem. Restricting our attention to a single elemental subproblem, $T \in \mathcal{T}_{h}$, we first re-write our local Lagrangian functional in a form suitable for applying the ideas developed for the energy bound. Every term other 
than the dissipative energy term, $\frac{\kappa}{2} \int_{T} \nabla w \cdot \nabla w \mathrm{~d} \Omega$, must not involve derivatives of $\hat{w}^{ \pm}$, which we can do in the present case by application of the Green's identity $-\int_{T} \nabla u \cdot \nabla w \mathrm{~d} \Omega=\int_{T} \Delta u w \mathrm{~d} \Omega-\int_{\partial T} \nabla u \cdot \mathbf{n} w \mathrm{~d} \Gamma$ to obtain the equivalent local Lagrangian functional

$$
\begin{aligned}
\mathcal{L}_{T}^{ \pm}\left(w^{ \pm} ; \pm \tilde{\psi}, \frac{\kappa}{2} \tilde{\lambda}^{u} \pm \tilde{\lambda}^{\psi}\right) \equiv \frac{\kappa}{2} \int_{T} \nabla w^{ \pm} \cdot \nabla w^{ \pm} \mathrm{d} \Omega \\
\quad-\frac{\kappa}{2}\left\{\int_{T}(f-\Delta \bar{u}) w^{ \pm} \mathrm{d} \Omega+\int_{\partial T}\left(\sigma_{T} \tilde{\lambda}^{u}+\nabla \bar{u} \cdot \mathbf{n}\right) w^{ \pm} \mathrm{d} \Gamma+\int_{T} f \bar{u} \mathrm{~d} \Omega\right\} \\
\mp\left\{\int_{T}\left(f^{\mathcal{O}}-\Delta \tilde{\psi}\right) w^{ \pm} \mathrm{d} \Omega+\int_{\partial T}\left(\sigma_{T} \tilde{\lambda}^{\psi}+\nabla \tilde{\psi} \cdot \mathbf{n}\right) w^{ \pm} \mathrm{d} \Gamma+\int_{T} f \tilde{\psi} \mathrm{d} \Omega\right\} .
\end{aligned}
$$

The functional we wish to minimize over $w^{ \pm}$can now be defined as

$$
J_{T}^{ \pm}\left(w^{ \pm}\right) \equiv \frac{\kappa}{2} \int_{T} \nabla w^{ \pm} \cdot \nabla w^{ \pm} \mathrm{d} \Omega-\int_{T} f^{ \pm} w^{ \pm} \mathrm{d} \Omega-\int_{\partial T} g^{ \pm} w^{ \pm} \mathrm{d} \Gamma,
$$

for $f^{ \pm} \equiv \frac{\kappa}{2}\{f-\Delta \bar{u}\} \pm\left\{f^{\mathcal{O}}-\Delta \tilde{\psi}\right\}$ and $g^{ \pm} \equiv \frac{\kappa}{2}\left\{\sigma_{T} \tilde{\lambda}^{u}+\nabla \bar{u} \cdot \mathbf{n}\right\} \pm\left\{\sigma_{T} \tilde{\lambda}^{\psi}+\nabla \tilde{\psi} \cdot \mathbf{n}\right\}$. Thus, the local relaxed primal minimization once again corresponds to a Poisson problem of the type represented in equation (1.1) with Neumann boundary conditions posed on a single element.

As was the case for the energy bound, we do not require, and in general cannot compute, the exact minimum of this local infinite-dimensional primal subproblem, but we can apply the same technique of dualizing this minimization problem in order to procure a computable lower bounding approximate to it.

Proposition 3.2. If we define the positive functional

$$
J_{T}^{c}(\mathbf{q}) \equiv \frac{1}{2} \int_{T} \mathbf{q} \cdot \mathbf{q} \mathrm{d} \Omega,
$$

where $\mathbf{q} \in \mathcal{H}(\operatorname{div} ; T)$, then we have

$$
J_{T}^{ \pm}\left(w^{ \pm}\right) \geq-\frac{1}{\kappa} J_{T}^{c}\left(\mathbf{q}^{ \pm}\right), \quad \forall w^{ \pm} \in \mathcal{H}^{1}(T), \forall \mathbf{q}^{ \pm} \in \mathcal{Q}^{ \pm}(T),
$$

for the set of functions

$$
\begin{aligned}
\mathcal{Q}^{ \pm}(T) \equiv\left\{\mathbf{q} \in \mathcal{H}(\operatorname{div} ; T) \mid \int_{T} \nabla \cdot \mathbf{q} v \mathrm{~d} \Omega-\int_{\partial T} \mathbf{q} \cdot \mathbf{n} v \mathrm{~d} \Gamma\right. \\
\left.\quad=-\int_{T} f^{ \pm} v \mathrm{~d} \Omega-\int_{\partial T} g^{ \pm} v \mathrm{~d} \Gamma, \forall v \in \mathcal{H}^{1}(T)\right\} .
\end{aligned}
$$

Proof. The local dual problem is derived as it was for the energy bound, but with modified data and the addition of the scaling parameter, $\kappa$. After expanding the positive expression for $\mathbf{q} \in \mathcal{Q}^{ \pm}(T)$

$$
\frac{1}{2 \kappa} \int_{T}\left(\mathbf{q}^{ \pm}-\kappa \nabla w\right)^{2} \mathrm{~d} \Omega \geq 0
$$

applying a Green's formula, and substituting the constraint from $\mathcal{Q}^{ \pm}(T)$, we obtain the expression

$$
\frac{1}{2 \kappa} \int_{T} \mathbf{q}^{ \pm} \cdot \mathbf{q}^{ \pm} \mathrm{d} \Omega+\frac{\kappa}{2} \int_{T} \nabla w^{ \pm} \cdot \nabla w^{ \pm} \mathrm{d} \Omega-\int_{T} f^{ \pm} w^{ \pm} \mathrm{d} \Omega-\int_{\partial T} g^{ \pm} w^{ \pm} \mathrm{d} \Gamma \geq 0 .
$$


Identifying $J_{T}^{ \pm}\left(w^{ \pm}\right)$and $J_{T}^{c}\left(\mathbf{q}^{ \pm}\right)$we arrive at the desired expression for the local lower bound.

As the functional $J_{T}^{ \pm}\left(w^{ \pm}\right)$only contains the terms from the Lagrangian that depended on $w^{ \pm}$, we must reintroduce the constant terms to secure the complete contributions from the local dual subproblems

$$
\mp s_{T}^{ \pm}=\int_{T} f\left(\frac{\kappa}{2} u_{h} \pm \psi_{h}\right) \mathrm{d} \Omega+\sup _{\mathbf{q}^{ \pm} \in \mathcal{Q}^{ \pm}(T)}-\frac{1}{\kappa} J_{T}^{c}\left(\mathbf{q}^{ \pm}\right) .
$$

3.3.1. Subproblem Approximation. Consider the splitting implied by the definition $\mathbf{q}_{h}=\kappa \nabla \bar{u}+\frac{\kappa}{2} \mathbf{q}_{h}^{u} \pm \mathbf{q}_{h}^{\psi}$. Propagation of this definition into the elemental subproblem reveals through the linearity of the gradient condition that indeed $\mathbf{q}_{h}^{u}$ and $\mathbf{q}_{h}^{\psi}$ can be computed independently. The resulting subproblems are

$$
\begin{aligned}
& \mathbf{q}_{h}^{u}=\arg \inf _{\mathbf{q}_{h} \in \mathcal{Q}_{h}^{u}(T)} J^{c}\left(\mathbf{q}_{h}\right), \\
& \mathbf{q}_{h}^{\psi}=\arg \inf _{\mathbf{q}_{h} \in \mathcal{Q}_{h}^{\psi}(T)} J^{c}\left(\mathbf{q}_{h}\right),
\end{aligned}
$$

for the dual feasible approximation sets

$$
\begin{gathered}
\mathcal{Q}_{h}^{u}(T) \equiv\left\{\mathbf{q} \in\left(\mathbb{P}^{q}(T)\right)^{d} \mid \int_{T} \nabla \cdot \mathbf{q} v \mathrm{~d} \Omega-\int_{\partial T} \mathbf{q} \cdot \mathbf{n} v \mathrm{~d} \Gamma=-\int_{T}\left(f+\Delta u_{h}\right) v \mathrm{~d} \Omega\right. \\
\left.-\int_{\partial T}\left(\sigma_{T} \lambda_{h}^{u}-\nabla u_{h} \cdot \mathbf{n}\right) v \mathrm{~d} \Gamma, \forall v \in \mathcal{H}^{1}(T)\right\}, \\
\mathcal{Q}_{h}^{\psi}(T) \equiv\left\{\mathbf{q} \in\left(\mathbb{P}^{q}(T)\right)^{d} \mid \int_{T} \nabla \cdot \mathbf{q} v \mathrm{~d} \Omega-\int_{\partial T} \mathbf{q} \cdot \mathbf{n} v \mathrm{~d} \Gamma=-\int_{T}\left(f^{\mathcal{O}}-\Delta \psi_{h}\right) v \mathrm{~d} \Omega\right. \\
\left.-\int_{\partial T}\left(\sigma_{T} \lambda_{h}^{\psi}+\nabla \psi_{h} \cdot \mathbf{n}\right) v \mathrm{~d} \Gamma, \forall v \in \mathcal{H}^{1}(T)\right\},
\end{gathered}
$$

in which we have again chosen $\bar{u}=u_{h}$ commensurate with our choice for the approximate multipliers. As the additional terms in the data of the dual feasibility constraint are just polynomial functions in the local finite element basis, there are no difficulties in choosing our dual approximation sets in this manner. The solvability of these subproblems is addressed by the following result.

Lemma 3.3. Suppose the forcing function $\left.f\right|_{T}$ and output function $\left.f^{\mathcal{O}}\right|_{T}$ are members of $\mathbb{P}^{r}(T)$, that $\lambda_{h}^{u}$ satisfies (3.6), and that $\lambda_{h}^{\psi}$ satisfies (3.7), then there exists at least one dual feasible function $\mathbf{q}_{h}^{u}$ that is a member of $\mathcal{Q}_{h}^{u}(T)$ and one dual feasible function $\mathbf{q}_{h}^{\psi}$ that is a member of $\mathcal{Q}_{h}^{\psi}(T)$, for $q \geq p$ and $q>r$.

Proof. Applying Green's formula to the $u_{h}$ Laplacian term in the constraint data for $\mathcal{Q}_{h}^{u}(T)$ of (3.17) and duplicating the proof of Lemma 2.3 with the resulting constraint data reveals the compatibility condition

$$
\int_{\partial T} \sigma_{T} \lambda_{h}^{u} \mathrm{~d} \Gamma=-\int_{T} f \mathrm{~d} \Omega
$$

which is satisfied by our choice for $\lambda_{h}^{u}$ as can be seen by choosing $\hat{v}=$ const on $T$ in the equilibration condition (3.6). The same argument holds for the adjoint dual subproblem, yielding the analogous compatibility condition

$$
\int_{\partial T} \sigma_{T} \lambda_{h}^{\psi} \mathrm{d} \Gamma=-\int_{T} f^{\mathcal{O}} \mathrm{d} \Omega
$$


for $f^{\mathcal{O}}$ and $\lambda_{h}^{\psi}$.

With the subproblem splitting just defined, the aggregated contributions to the upper and lower bounds become

$$
\begin{aligned}
s_{h}^{ \pm}=\mp & \int_{\Omega} f\left(\frac{\kappa}{2} u_{h} \pm \psi_{h}\right) \mathrm{d} \Omega \pm \frac{1}{\kappa} J^{c}\left(\kappa \nabla u_{h}+\frac{\kappa}{2} \hat{\mathbf{q}}_{h}^{u} \pm \hat{\mathbf{q}}_{h}^{\psi}\right) \\
=\mp & \int_{\Omega} f\left(\frac{\kappa}{2} u_{h} \pm \psi_{h}\right) \mathrm{d} \Omega \pm \frac{\kappa}{2} \int_{\Omega} \nabla u_{h} \cdot \nabla u_{h} \mathrm{~d} \Omega+\int_{\Omega}\left(\frac{\kappa}{2} \hat{\mathbf{q}}_{h}^{u} \pm \hat{\mathbf{q}}_{h}^{\psi}\right) \cdot \nabla u_{h} \mathrm{~d} \Omega \\
& \quad+\frac{1}{2} \int_{\Omega} \hat{\mathbf{q}}_{h}^{u} \cdot \hat{\mathbf{q}}_{h}^{\psi} \mathrm{d} \Omega \pm \frac{\kappa}{4} J^{c}\left(\hat{\mathbf{q}}_{h}^{u}\right) \pm \frac{1}{\kappa} J^{c}\left(\hat{\mathbf{q}}_{h}^{\psi}\right) \\
= & -\int_{\Omega} f \psi_{h} \mathrm{~d} \Omega+\frac{1}{2} \int_{\Omega} \hat{\mathbf{q}}_{h}^{u} \cdot \hat{\mathbf{q}}_{h}^{\psi} \mathrm{d} \Omega \pm \frac{\kappa}{4} J^{c}\left(\hat{\mathbf{q}}_{h}^{u}\right) \pm \frac{1}{\kappa} J^{c}\left(\hat{\mathbf{q}}_{h}^{\psi}\right),
\end{aligned}
$$

in which we have invoked (3.4) with $v=u_{h}$ as well as used orthogonality relationships analogous to that proved in Lemma 2.4 .

3.4. Output Bound Procedure. The introduction of the scaling parameter $\kappa$ allows us to optimize the sharpness of the computed bounds in addition to providing dimensional consistency. From the previous section we have the expression for the upper and lower output bounds

$$
s_{h}^{ \pm}=\bar{s}_{h} \pm \kappa z_{h}^{u} \pm \frac{1}{\kappa} z_{h}^{\psi},
$$

where

$$
\bar{s}_{h}=\frac{1}{2} \int_{\Omega} \hat{\mathbf{q}}_{h}^{u} \cdot \hat{\mathbf{q}}_{h}^{\psi} \mathrm{d} \Omega-\int_{\Omega} f \psi_{h} \mathrm{~d} \Omega, \quad z_{h}^{u}=\frac{1}{4} J^{c}\left(\hat{\mathbf{q}}_{h}^{u}\right), \quad z_{h}^{\psi}=J^{c}\left(\hat{\mathbf{q}}_{h}^{\psi}\right),
$$

Maximizing the lower bound and minimizing the upper bound with respect to $\kappa$ yields the optimal value $\kappa^{2}=z_{h}^{\psi} / z_{h}^{u}$.

The complete method with optimal scaling for upper and lower bounds on linear functional outputs can now be written as three steps:

1. Global Approximation:

Find $u_{h} \in \mathcal{U}_{h}$ such that

$$
\int_{\Omega} \nabla u_{h} \cdot \nabla v \mathrm{~d} \Omega=\int_{\Omega} f v \mathrm{~d} \Omega, \quad \forall v \in \mathcal{U}_{h}
$$

and find $\psi_{h} \in \mathcal{U}_{h}$ such that

$$
\int_{\Omega} \nabla v \cdot \nabla \psi_{h} \mathrm{~d} \Omega=-\int_{\Omega} f^{\mathcal{O}} v \mathrm{~d} \Omega, \quad \forall v \in \mathcal{U}_{h} .
$$

2. Global Equilibration:

Find $\lambda_{h}^{u} \in \Lambda_{h}$ such that

$$
\sum_{T \in \mathcal{T}_{h}} \int_{\partial T} \sigma_{T} \lambda_{h}^{u} \hat{v} \mathrm{~d} \Gamma=\int_{\Omega} \nabla u_{h} \cdot \nabla \hat{v} \mathrm{~d} \Omega-\int_{\Omega} f \hat{v} \mathrm{~d} \Omega, \quad \forall \hat{v} \in \hat{\mathcal{U}}_{h},
$$

and find $\lambda_{h}^{\psi} \in \Lambda_{h}$ such that

$$
\sum_{T \in \mathcal{T}_{h}} \int_{\partial T} \sigma_{T} \lambda_{h}^{\psi} \hat{v} \mathrm{~d} \Gamma=-\int_{\Omega} f \hat{v} \mathrm{~d} \Omega-\int_{\Omega} \nabla \hat{v} \cdot \nabla \psi_{h} \mathrm{~d} \Omega, \quad \forall \hat{v} \in \hat{\mathcal{U}}_{h}
$$


3. Local Dual Subproblems:

Find $\hat{\mathbf{q}}_{h}^{u}$ such that

$$
\hat{\mathbf{q}}_{h}^{u}=\arg \inf _{\hat{\mathbf{q}}_{h} \in \hat{\mathcal{Q}}_{h}^{u}} J^{c}\left(\hat{\mathbf{q}}_{h}\right),
$$

find $\hat{\mathbf{q}}_{h}^{\psi}$ such that

$$
\hat{\mathbf{q}}_{h}^{\psi}=\arg \inf _{\hat{\mathbf{q}}_{h} \in \hat{\mathcal{Q}}_{h}^{\psi}} J^{c}\left(\hat{\mathbf{q}}_{h}\right),
$$

and, from equation (3.20) and the optimal $\kappa$, calculate

$$
s_{h}^{ \pm}=\bar{s}_{h} \pm 2 \sqrt{z_{h}^{u} z_{h}^{\psi}} .
$$

The local dual subproblems for the output bounds can be solved in the same manner as the local energy dual subproblems. The important point being that once the finite element approximations $u_{h}$ and $\psi_{h}$ have been computed, the solutions can be equilibrated and quantitative bounds computed on the exact output to the infinitedimensional continuum equation with asymptotically linear cost in the size of the finite element discretization and in parallel. In addition, the elemental contribution to the bound gap, $\frac{\kappa}{4} J_{T}^{c}\left(\mathbf{q}_{h}^{u}\right)+\frac{1}{\kappa} J_{T}^{c}\left(\mathbf{q}_{h}^{\psi}\right)$, can serve as an informative mesh adaptivity indicator for controlling the error in the output, as was done in [15] for a two-level error bound method and in [3] for an asymptotic error estimation method.

3.4.1. Properties of the Output Bounds. The upper and lower bounding properties are direct consequences of the saddle point property of the relaxed constrained minimization reformulation (3.2) and the local dual property of Proposition 3.2. The following proposition addresses the accuracy of the computed bounds by showing that the bounds will converge at the optimal rate when both the primal and adjoint finite element approximations are in the asymptotic convergence regime.

Proposition 3.4. Suppose that $u_{h}, \psi_{h}, \lambda_{h}^{u}$, and $\lambda_{h}^{\psi}$ are solutions of the above finite element approximation problems and equilibration problems, then

$$
\begin{aligned}
& s-s_{h}^{-} \leq C\left|u-u_{h}\right|_{1}\left|\psi-\psi_{h}\right|_{1}, \\
& s_{h}^{+}-s \leq C\left|u-u_{h}\right|_{1}\left|\psi-\psi_{h}\right|_{1} .
\end{aligned}
$$

Proof. Applying the definitions from the procedure, we know that the lower a posteriori bound, for instance, itself has the bound $s-s_{h}^{-} \leq s_{h}^{+}-s_{h}^{-}=2 \sqrt{z_{h}^{u} z_{h}^{\psi}}$. The arguments of Lemma 2.5 can be applied to the $z_{h}^{u}$ and $z_{h}^{\psi}$ factors to show that they are bounded by $C\left|u-u_{h}\right|_{1}^{2}$ and $C\left|\psi-\psi_{h}\right|_{1}^{2}$, respectively.

4. Numerical Results. We verify the method numerically for three cases: constant forcing on the unit square, linear forcing on the unit square, and zero forcing on an L-shaped domain with a corner singularity. Linear finite elements, $p=1$, and quadratic subproblems, $q=2$, are employed with the domain average output $s=\int_{\Omega} f^{\mathcal{O}} u \mathrm{~d} \Omega$, where $f^{\mathcal{O}}=$ const, for all cases.

All three cases have analytically exact solutions with which we are able to verify the method and calculate the effectivities of the bounds,

$$
\theta^{ \pm}=\frac{\left|s-s_{h}^{ \pm}\right|}{\left|s-s_{h}\right|}
$$

which indicate the sharpness by comparing the error in the bounds to the error in the finite element approximation. 


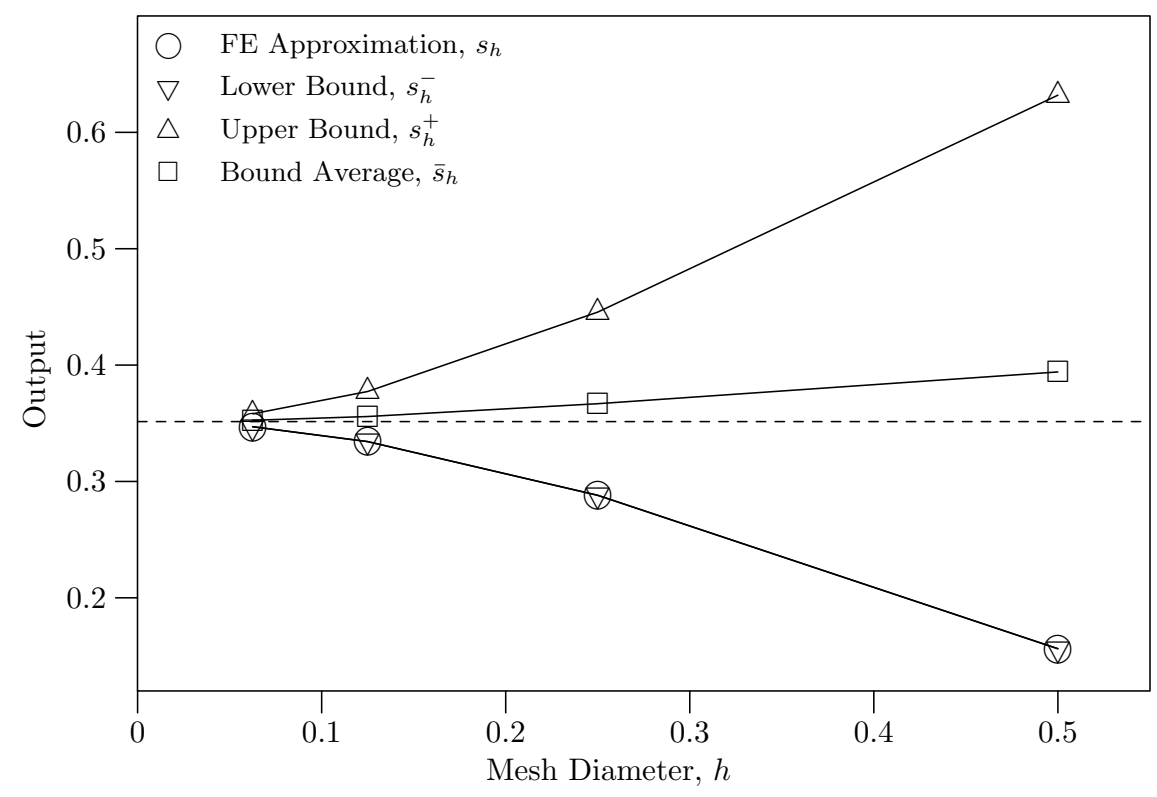

FIG. 4.1. Uniformly forced square domain.

4.1. Uniformly Forced Square Domain. The first case is a uniformly forced unit square domain with $f=f^{\mathcal{O}}=\sqrt{10}$. The analytical solution is given by

$$
u(x, y)=\frac{16 \sqrt{10}}{\pi^{4}} \sum_{\text {odd } i, j=1}^{\infty} \frac{(-1)^{(i+j) / 2-1}}{i j\left(i^{2}+j^{2}\right)} \cos \left(i \frac{\pi}{2} x\right) \cos \left(j \frac{\pi}{2} y\right),
$$

This case is special in that the forcing and output are identical and the boundary data is homogeneous, leading to primal and adjoint problem data which differ by only a sign. It is well known that for this special case, called compliance, the finite element approximation for the output is a lower bound. The numerical results given in Table 4.1 demonstrate that our method, while more expensive, does no worse than the inherent bound for this special case. The results for both the finite element approximation and the output bounds asymptotically approach the optimal finite element convergence rate of $O\left(h^{2}\right)$. This example also evinces that the bound average, $\bar{s}_{h}$, can sometimes be a more accurate output approximation than that from the finite element approximation.

\begin{tabular}{|c||c|ccc|cc|}
\hline$h$ & $s_{h}$ & $s_{-}$ & $\bar{s}_{h}$ & $s^{+}$ & $\theta^{-}$ & $\theta^{+}$ \\
\hline \hline$\frac{1}{2}$ & 0.156 & 0.156 & 0.394 & 0.632 & 1.0 & 1.4 \\
$\frac{1}{4}$ & 0.288 & 0.288 & 0.367 & 0.446 & 1.0 & 1.5 \\
$\frac{1}{8}$ & 0.334 & 0.334 & 0.356 & 0.377 & 1.0 & 1.5 \\
$\frac{1}{16}$ & 0.347 & 0.347 & 0.353 & 0.358 & 1.0 & 1.5 \\
\hline \multicolumn{5}{|c}{ TABLE 4.1} \\
\hline
\end{tabular}

Numerical results for the uniformly forced square domain test case for which $s=0.351$. 


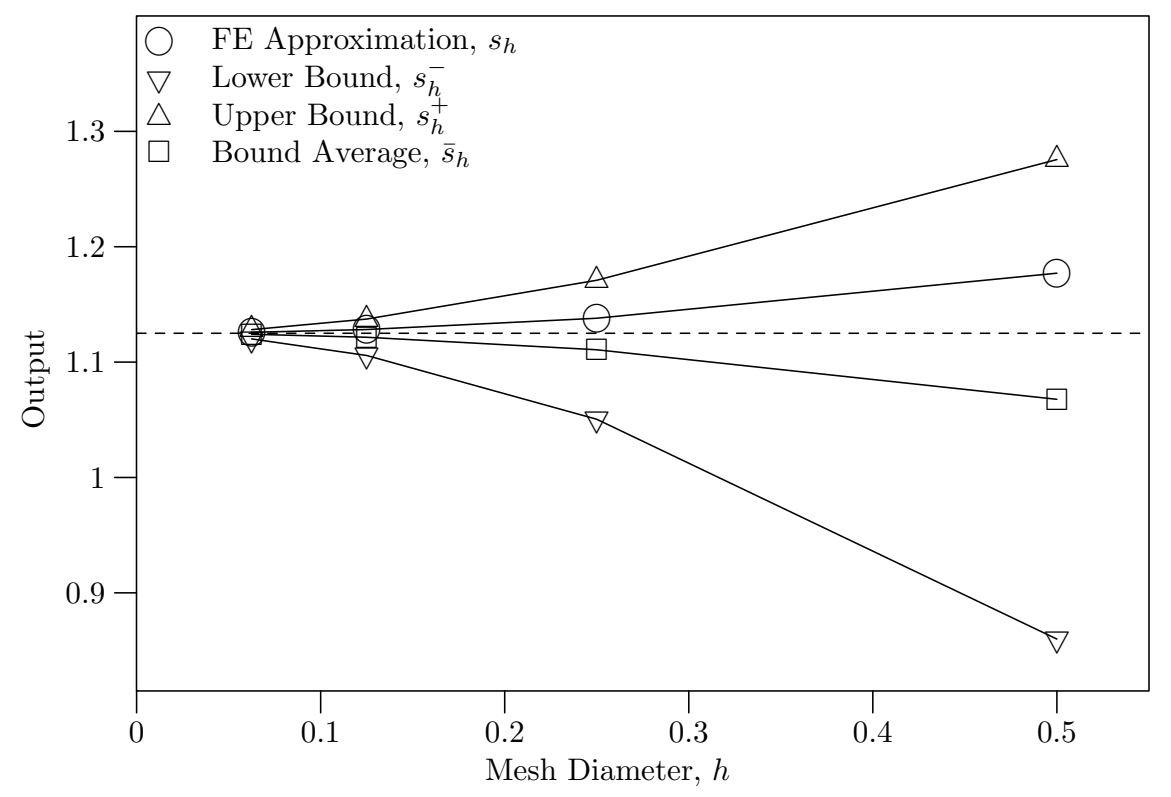

FIG. 4.2. Linearly forced square domain.

4.2. Linearly Forced Square Domain. The second case is a linearly forced square domain with $f^{\mathcal{O}}=1$, and the forcing and nonhomogeneous boundary conditions chosen to produce the exact solution

$$
u(x, y)=\frac{3}{2} y^{2}(1-y)+4 x y .
$$

As this test case is not a special case, the convergence histories of Table 4.2 and Figure 4.2 depict the more general situation in which none of the computed quantities coincide. Whereas in the first example we saw that the bound average can possibly be a more accurate output approximation than the finite element approximation, in this example we see that this is definitely not always true since the finite element approximation for the output is $0.5 \%$ better. As for the first example, the results for both the finite element approximation and the output bounds asymptotically approach the optimal finite element convergence rate of $O\left(h^{2}\right)$.

\begin{tabular}{|c||c|ccc|cc|}
\hline$h$ & $s_{h}$ & $s_{-}$ & $\bar{s}_{h}$ & $s^{+}$ & $\theta^{-}$ & $\theta^{+}$ \\
\hline \hline$\frac{1}{2}$ & 1.177 & 0.860 & 1.068 & 1.276 & 5.1 & 2.9 \\
$\frac{1}{4}$ & 1.138 & 1.050 & 1.111 & 1.171 & 5.7 & 3.5 \\
$\frac{1}{8}$ & 1.128 & 1.106 & 1.212 & 1.137 & 5.9 & 3.8 \\
$\frac{1}{16}$ & 1.126 & 1.120 & 1.124 & 1.128 & 6.0 & 3.8 \\
\hline
\end{tabular}

Numerical results for the linearly forced square domain test case for which $s=1.125$. 


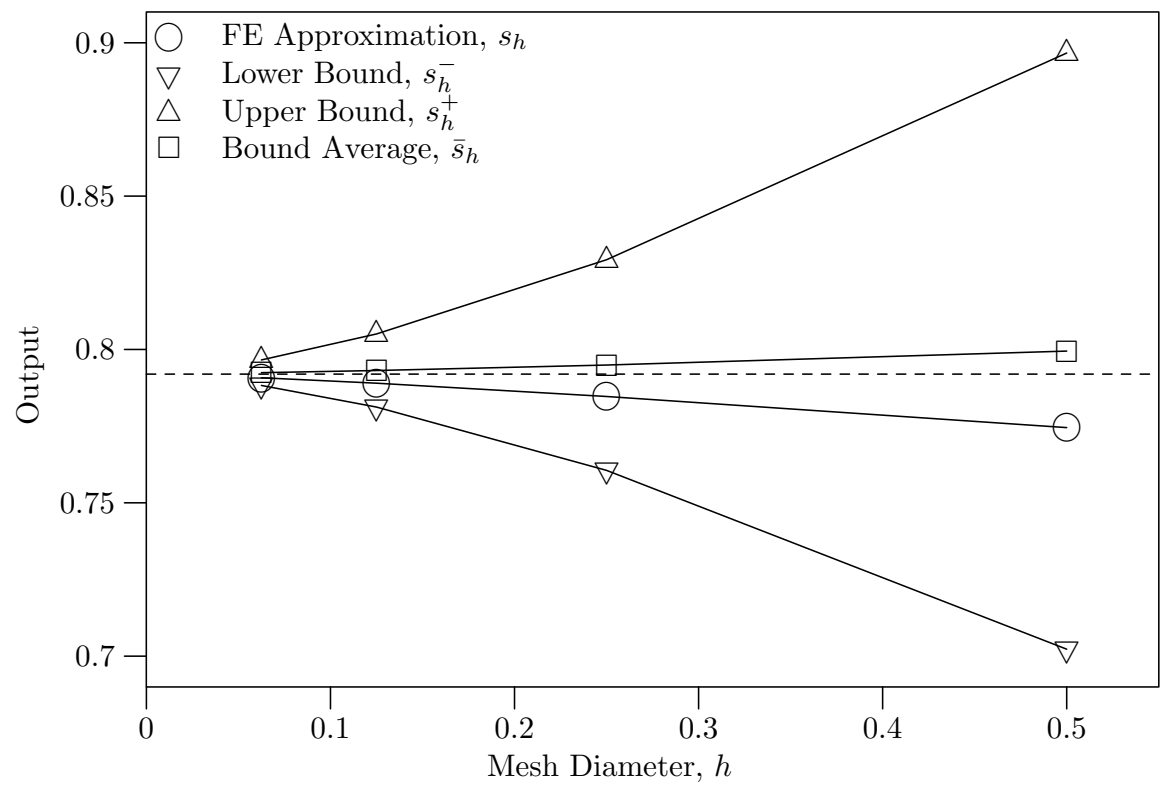

FIG. 4.3. Unforced corner domain.

4.3. Unforced Corner Domain. Last, we consider the Laplace equation on a nonconvex domain with $f^{\mathcal{O}}=1$. The domain is the standard L-shaped domain with a reentrant corner that results from removing the lower right quadrant of the unit square. The Dirichlet boundary conditions were chosen to produce the solution

$$
u(r, \phi)=r^{\frac{2}{3}} \sin \frac{2}{3} \phi,
$$

where the distance from the corner point is $r(x, y)=\left\{(x-1 / 2)^{2}+(y-1 / 2)^{2}\right\}^{\frac{1}{2}}$ and the angle from the upper surface of the corner is $\phi(x, y)=\arctan \left(\frac{y-1 / 2}{x-1 / 2}\right)$.

In this example we demonstrate that the bounds are valid even for problems with singularities. As can be seen from Table 4.3 and Figure 4.3, the results for both the finite element approximation and the output bounds asymptotically approach the optimal finite element convergence rate of $O\left(h^{\frac{4}{3}}\right)$ for elliptic problems posed on a domain with right-angled reentrant corner [18]. Once again we see that the bound average has the potential to be a better output approximation than the finite element method.

\begin{tabular}{|c||c|ccc|cc|}
\hline$h$ & $s_{h}$ & $s_{-}$ & $\bar{s}_{h}$ & $s^{+}$ & $\theta^{-}$ & $\theta^{+}$ \\
\hline \hline$\frac{1}{2}$ & 0.775 & 0.702 & 0.799 & 0.897 & 5.1 & 6.0 \\
$\frac{1}{4}$ & 0.785 & 0.761 & 0.795 & 0.829 & 4.3 & 5.1 \\
$\frac{1}{8}$ & 0.789 & 0.781 & 0.793 & 0.805 & 3.6 & 4.4 \\
$\frac{1}{16}$ & 0.791 & 0.788 & 0.792 & 0.797 & 3.1 & 3.9 \\
\hline \multicolumn{5}{|c}{ TABLE 4.3} \\
\hline
\end{tabular}

Numerical results for the unforced corner domain test case for which $s=0.792$.

We close by demonstrating the use of the previously introduced elemental contributions to the bound gap, $\Delta_{T} \equiv \frac{\kappa}{4} J_{T}^{c}\left(\mathbf{q}_{h}^{u}\right)+\frac{1}{\kappa} J_{T}^{c}\left(\mathbf{q}_{h}^{\psi}\right)$, as a mesh adaptivity indicator 
for controlling the error in the output. Figure 4.4 displays the solution field, adjoint field, intermediate local error indicators, and final adaptive mesh that results from preferentially refining elements with relatively high contributions to the bound gap. We initiated the adaptive process on a uniform mesh of 6 elements and adaptively refined until the output uncertainty was less than 0.005 , that is, until we had a certificate of precision at least as good as $s=s_{h} \pm 0.005$. The adaptive refinement process met this target by producing the certificate of precision $s=0.791 \pm 0.00456$ using 1167 elements. Achieving the same certainty with uniform refinement requires 6144 elements.
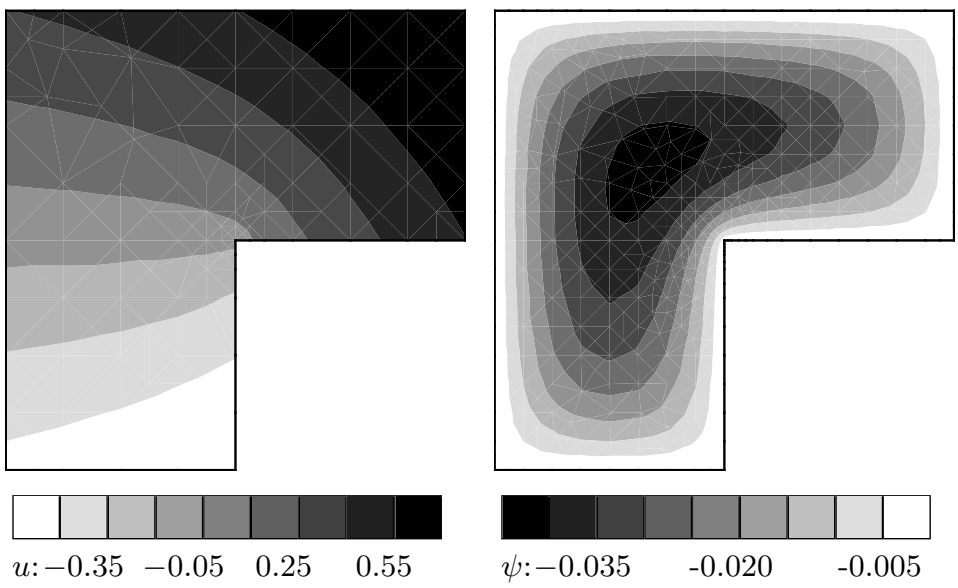

(a) Adaptive solution field.

(b) Adaptive adjoint field.

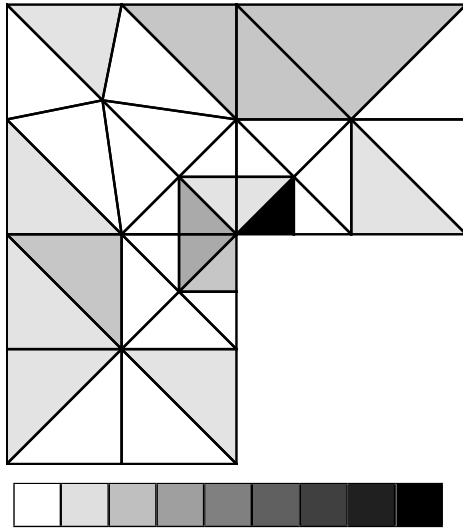

$\Delta_{T}: 0.02 \quad 0.06 \quad 0.10 \quad 0.14$

(c) Intermediate local error indicators.

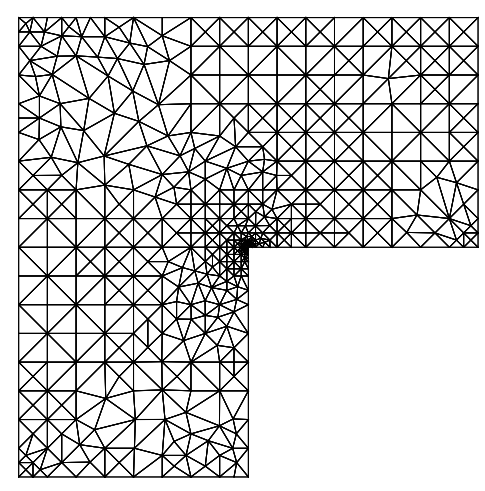

(d) Final adaptive mesh.

FIG. 4.4. Unforced corner domain adaptive solutions, local indicators and meshes. 
Acknowledgments. We would like to acknowledge our long-standing and fecund collaboration with Professor A. T. Patera at MIT. A. M. Sauer-Budge and J. Peraire gratefully acknowledge the support for this work provided by the Singapore-MIT Alliance.

\section{REFERENCES}

[1] M. Ainsworth and J. T. Oden, A unified apporach to a posteriori error estimation using element residual methods, Numerische Mathematik, (1993), pp. 23-50.

$[2]-$ A posteriori error estimation in finite element analysis, Computer Methods in Applied Mechanics and Engineering, (1997), pp. 1-88.

[3] R. BECKER AND R. RANNACHER, A feedback approach to error control in finite element method: Basic analysis and examples, East - West Journal of Numerical Mathematics, 4 (1996), pp. 237-264.

[4] F. Brezzi And M. Fortin, Mixed and Hybrid Finite Element Methods, Springer Series in Computational Mathematics, Springer-Verlag, 1991.

[5] T. CaO, D. W. Kelly, and I. H. SloAn, Local error bounds for post-processed finite element calculations, International Journal for Numerical Methods in Engineering, 45 (1999), pp. 1085-1098.

[6] P. Destuynder AND B. MÉtivet, Explicit error bounds in a conforming finite element method, Mathematics of Computation, 68 (1999), pp. 1379-1396.

[7] B. Fraeiss De Veubeke, Displacement and equilibrium models in the finite element method, in B.M. Fraeijs de Veubeke Memorial Volume of Selected Papers, M. geradin, ed., Sijthoff and Noordhoff International Publishers, 1980.

[8] A. Jameson, Aerodynamic design via control theory, Journal of Scientific Computing, 3 (1988), pp. 233-260.

[9] P. Ladevèze and D. Leguillon, Error estimate procedure in the finite element method and applications, SIAM Journal of Numerical Analysis, 20 (1983), pp. 485-509.

[10] P. LAdevèze AND P. Rougeot, New advances on a posteriori error on constitutive relation in f.e. analysis, Computer Methods in Applied Mechanics and Engineering Computer Methods in Applied Mechanics and Engineering Computer Methods in Applied Mechanics and Engineering, 150 (1997), pp. 239-249.

[11] Y. Maday And A. T. PATERA, Numerical analysis of a posteriori finite element bounds for linear functional outputs, Mathematical Models and Methods in Applied Sciences, 10 (2000), pp. $785-799$.

[12] P. Morin, R. H. Nochetto, and K. G. Siebert, Convergence of adaptive finite element methods, SIAM Review, 44 (2002), pp. 631-658.

[13] M. Paraschivoiu And A. T. Patera, A hierarchical duality approach to bounds for the outputs of partial differential equations, Computer Methods in Applied Mechanics and Engineering, 158 (1998), pp. 389-407.

[14] M. Paraschivoiu, J. Peraire, and A. T. Patera, A posteriori finite element bounds for linear-functional outputs of elliptic partial differential equations, Computer Methods in Applied Mechanics and Engineering, 150 (1997), pp. 289-312.

[15] J. Peraire And A. Patera, Bounds for linear-functional outputs of coercive partial differential equations: Local indicators and adaptive refinement, in Proceedings of the Workshop On New Advances in Adaptive Computational Methods in Mechanics, P. Ladeveze and J. Oden, eds., Cachan, September 17-19 1997, Elsevier.

[16] A. Quarteroni And A. Valli, Numerical Approximation of Partial Differential Equations, Springer Series in Computational Mathematics, Springer, 1997.

[17] A. M. Sauer-Budge and J. Peraire, Computing bounds for linear functional outputs of exact weak solutions to the advection-diffusion-reaction equation. In preparation.

[18] G. Strang And G. J. Fix, An Analysis of the Finite Element Method, Prentice-Hall, 1973. 